Invariant measures for the n-dimensional border collision normal form

Glendinning, Paul

2014

MIMS EPrint: 2015.10

Manchester Institute for Mathematical Sciences

School of Mathematics

The University of Manchester

\footnotetext{
Reports available from: http://eprints.maths.manchester.ac.uk/

And by contacting: The MIMS Secretary

School of Mathematics

The University of Manchester

Manchester, M13 9PL, UK
} 


\title{
Invariant measures for the n-dimensional border collision normal form
}

\author{
Paul Glendinning \\ School of Mathematics, University of Manchester, \\ Manchester M13 9PL, U.K. \\ p.a.glendinning@manchester.ac.uk \\ Received (to be inserted by publisher)
}

\begin{abstract}
The border collision normal form is a continuous piecewise affine map of $\mathbb{R}^{n}$ with applications in piecewise smooth bifurcation theory. We show that these maps have absolutely continuous invariant measures for an open set of parameter space and hence that the attractors have Hausdorff (fractal) dimension $n$. If $n=2$ the attractors have topological dimension two, i.e. they contain open sets, and if $n>2$ then they have topological dimension $n$ generically.
\end{abstract}

Keywords: border collision bifurcation, attractor, piecewise smooth systems, piecewise affine systems

\section{Introduction}

Continuous, piecewise affine maps have been studied by a variety of different research communities. Gardini and co-workers have studied them theoretically and numerically as canonical examples of endomorphisms of the plane [Gardini, 1992; Mira, Gardini et al., 1996; Mira, Rauzy et al., 1996]. The Lozi map and extensions are two dimensional maps for which it is possible to prove the existence of strange attractors with absolutely continuous invariant measures on unstable manifolds, i.e. the attractors have a fractal structure with a nice one-dimensional projection [Lozi, 1978; Misiurewicz, 1980; Young, 1985]. More recently, interest in digital control methods and electronic circuits has led to their study as a normal form, called the border collision normal form, for the situation that arises when a fixed point strikes the boundary between regions in which different maps are applied with continuity across the boundary [Banerjee \& Grebogi, 1999; Banerjee, Ranjan \& Grebogi, 2000; Banerjee, Yorke \& Grebogi, 1998; di Bernardo et al., 2008; Nusse et al., 1994; Nusse \& Yorke, 1992; Simpson, 2010; Simpson \& Meiss, 2010]. They also arise in economic models [Bischi et al., 2000; Cánovas \& Lineros, 2001] and a further, more abstract set of results has been developed in the general study of piecewise affine maps allowing discontinuities [Buzzi, 1999, 2001; Buzzi \& Keller, 2001; Ishii \& Sands, 2007; Tsujii, 2001].

Numerical simulations of the border collision normal form in dimension two suggest that for certain parameters attractors appear to be (topologically) two-dimensional regions in the plane, i.e. two-dimensional regions on which the map is transitive and periodic orbits are dense. However, proofs of the existence of these regions are either restricted to cases where the dynamics can be analyzed by related one-dimensional maps [Bischi et al., 2000], or to a specially chosen countable set of parameters at which there is a finite Markov partition with sufficient expansion in the map [Glendinning \& Wong, 2011], see also [Dobrynskii, 1998; Dobrynskiy, 1999]. Glendinning and Jeffrey [2012] show that analogous attractors exist in the $n$ - 
dimensional version of the border collision normal form due to di Bernardo [di Bernardo, 2003]. They use this, and a connection established between the border collision normal form and return maps of grazingsliding bifurcations of flows, to argue that bifurcation theorems for piecewise smooth systems may be necessarily more complicated than local bifurcation theorems for smooth systems since results are dependent on the dimension of the ambient phase space. The multi-dimensional results here add weight to this argument for a curse of dimensionality in piecewise smooth bifurcation theory as we show that there are open sets of parameters for the existence of attractors of the $n$-dimensional normal form that cannot arise as attractors of the $(n-1)$-dimensional normal form.

One of the main results of this paper is that attractors with topological dimension two exist for open sets of parameters in the two dimensional normal form, and these results are extended to higher dimensions where attractors with Hausdorff (or fractal) dimension $n$ are shown to exist for open sets of parameters for the border collision normal form in $\mathbb{R}^{n}$ and that generically these also have topological dimension $n$. To do this we move from a topological description of chaos to an ergodic description using invariant measures (i.e. a probabilistic interpretation of the chaotic dynamics). Invariant measures act rather like probability distributions for the deterministic dynamics, so integration with respect to this measure is equivalent (for 'most' orbits) to taking time averages. In this way invariant measures provide a mathematical description of the more stochastic features of deterministic chaos. The dimension of an invariant measure is the (fractal) dimension of the smallest invariant set such that all sets outside the invariant set have measure zero, so this is a natural attractor to consider under the measure. Intuitively, a measure $\mu$ is absolutely continuous with respect to the standard Lebesgue meaure if there is an integrable function $\phi$ that is the equivalent of a probability density function.

Provided some expansion properties hold, results of Tsujii [2001] and Buzzi [1999, 2001] can be used to establish the existence of an invariant measure which is absolutely continuous with respect to the standard Lebesgue measure on $\mathbb{R}^{n}$, which implies the attractor (or possibly attractors) has Hausdorff dimension $n$, and a further result of Buzzi shows that the attractor has non-empty interior and hence topological dimension $n$ always if $n=2$ and generically if $n>2$ [Buzzi, 2001].

In terms of coordinates $z=\left(x_{1}, \ldots, x_{n}\right)^{T}$ in $\mathbb{R}^{n}$ a border-collision map is

$$
z_{n+1}=F\left(z_{n}\right)= \begin{cases}A_{0} z_{k}+m & \text { if } x_{1} \leq 0 \\ A_{1} z_{k}+m & \text { if } x_{1} \geq 0\end{cases}
$$

where $A_{0}$ and $A_{1}$ are constant matrices, $m$ is a constant vector, and the system is continuous across the surface $x_{1}=0$. For generic choices of $A_{0}$ and $A_{1}$ an affine change of coordinates can be used to transform the matrices into observer canonical form [di Bernardo, 2003; di Bernardo et al., 2011; Nusse \& Yorke, 1992 ] and the vector $m$ to a vector that is zero apart from the first coordinate. In two dimensions this means we can (generically) take

$$
A_{\alpha}=\left(\begin{array}{cc}
T_{\alpha} & 1 \\
-D_{\alpha} & 0
\end{array}\right), \quad m=\left(\begin{array}{c}
\mu \\
0
\end{array}\right)
$$

for $\alpha=0,1$. The constants $T_{\alpha}$ and $D_{\alpha}$ have natural interpretations as the trace and determinant of the matrices, and by a change of scale only the sign of $\mu$ matters if $\mu \neq 0$. Moreover, by exchanging the roles of the left and right variables, $\mu=-1$ can be transformed to $\mu=1$, so we can work with $\mu=1$ in our examples. In higher dimensions [di Bernardo, 2003; di Bernardo et al., 2011] the observer canonical form is given by $(21)$ and $m=(\mu, 0, \ldots, 0)^{T}$. With these choices (1) is called the border collision normal form.

The theoretical results we use depend on each of the maps (subscripts 0 and 1) being expanding, i.e. $\|F(x)-F(y)\|>k\|x-y\|$ for some $k>1$ for all $x$ and $y$ in $x_{1} \geq 0$ and for all $x$ and $y$ in $x_{1} \leq 0$. We will refer to these as piecewise expanding maps, or piecewise linear expanding maps if the maps are all linear or affine. For affine maps with linear part $A$ a map is expanding in the standard Euclidean metric if the eigenvalues of the symmetric matrix $A^{T} A$ lie outside the unit circle. Unfortunately this is not the case for the maps considered here since the condition is equivalent to $T_{\alpha}^{2}<0$. However, by using an equivalent metric (used in [Glendinning, 2014] for the contracting case and [Barnsley et al., 1989] for Iterated Function Systems) we are able to find a metric in which the affine maps can be expanding, and hence the results of 
Tsujii [2001] and Buzzi [2001] can be used to establish the existence of an absolutely continuous invariant measure and, generically, an attractor with non-empty interior.

Theorem 1. Consider the border collision normal form in $\mathbb{R}^{n}(n=2,3, \ldots)$ with $\mu=1$. Then there exists an open region of parameter space $B_{n}$ bounded by a finite set of closed curve segments such that for each parameter in $B_{n}$ the border collision normal form has at least one attractor with an invariant measure absolutely continuous with respect to $n$-dimensional Lebesgue measure. If $n=2$ then this attractor has topological dimension equal to two (i.e. non-empty interior), whilst if $n>2$ then it has Hausdorff dimension equal to $n$ and generically has topological dimension equal to $n$.

Explicit regions of parameter space are identified in the proof. The new results of this paper are that the theorems of Tsujii and Buzzi can be applied to the border collision normal form in arbitrary dimension using the equivalent metric of section two and its generalization defined in section five. This is important as it provides more information about the structure of attractors in these systems that are important for applications, but there is no claim that this improves or augments the understanding of abstract piecewise affine systems.

The remainder of this paper is organized as follows. In section two we describe the equivalent metric in $\mathbb{R}^{2}$ and give conditions which imply that the border collision normal form is piecewise expanding in this metric. In section three we recall the results of Tsujii [2001] and Buzzi [1999,2001]. These results make it possible to show that if the border collision normal form has an invariant polyhedral region and the conditions of section two hold then the border collision normal form has an absolutely continuous invariant measure. In section four we show that these conditions are satisfied on an open set of parameters, completing the proof of the stated theorem in two dimensions. In section five we show that the existence of an absolutely contiuous invariant measure for open sets of parameters can be proved for the border collision normal form in $\mathbb{R}^{n}, n>2$, and that generically the topological demension is also $n$.

\section{Expansion and Equivalent metrics}

We say that a differentiable map $f$ is expanding on $U \subset \mathbb{R}^{n}$ in the metric $\|$.$\| iff$

$$
\frac{\|D f(x) v\|}{\|v\|}=\lambda>1
$$

for all $x \in U$ and $v \in \mathbb{R}^{n}$ (or more accurately the tangent space at $x$ ). For the remainder of this section we work in the plane, $n=2$.

As noted earlier, neither of the maps defining the border collision normal form is expanding in the Euclidean metric, but there is an equivalent metric for which the piecewise affine maps may be expanding.

Recall that a metric $d$ is equivalent to the Euclidean metric $\|\cdot\|_{2}$ on $\mathbb{R}^{2}$ iff there exists strictly positive constants $c_{1}<c_{2}$ such that

for all $x, y \in \mathbb{R}^{2}$.

$$
c_{1}\|x-y\|_{2} \leq d(x, y) \leq c_{2}\|x-y\|_{2}
$$

In the border collision normal form (1) the derivative (or Jacobian) of the map takes the form

$$
D F(x)=J=\left(\begin{array}{cc}
t & 1 \\
-d & 0
\end{array}\right)
$$

and if $|$.$| is the standard one-dimensional Euclidean distance, and \alpha>0$ then in the metric $d_{\alpha}$ defined by

$$
d_{\alpha}(x, y)=\alpha\left|x_{1}-y_{1}\right|+\left|x_{2}-y_{2}\right|
$$

the distance between images is

$$
d_{\alpha}(J x, J y)=\alpha\left|t x_{1}+x_{2}-\left(t y_{1}+y_{2}\right)\right|+\left|d x_{1}-d y_{1}\right| .
$$

We will establish conditions on $\alpha, t$ and $d$ under which this is an expanding map using the simple inequality $|a+b| \geq|a|-|b|$, so (6) becomes

$$
d_{\alpha}(J x, J y)=\left|t\left(x_{1}-y_{1}\right)+\left(x_{2}-y_{2}\right)\right| \geq\left|x_{2}-y_{2}\right|-|t|\left|x_{1}-y_{1}\right|
$$


and hence

$$
\begin{aligned}
d_{\alpha}(J x, J y) & \geq \alpha\left(\left|x_{2}-y_{2}\right|-|t|\left|x_{1}-y_{1}\right|\right)+\left|d x_{1}-d y_{1}\right| \\
& \geq \alpha\left(\left(\alpha^{-1}|d|-|t|\right)\left|x_{1}-y_{1}\right|+\left|x_{2}-y_{2}\right|\right) .
\end{aligned}
$$

Thus

$$
\left(\alpha^{-1}|d|-|t|\right) \geq \alpha
$$

implies that

$$
d_{\alpha}(J x, J y) \geq \alpha\left(\alpha\left|x_{1}-y_{1}\right|+\left|x_{2}-y_{2}\right|\right)=\alpha d_{\alpha}(x, y) .
$$

and so if (8) holds for some $\alpha>1$ then the map is expanding. Inequality (8), with $\alpha>0$ is equivalent to

$$
\alpha^{2}+|t| \alpha-|d|<0
$$

and this has solutions in $\alpha>1$ if

$$
|d|>1+|t|
$$

Thus if

$$
\left|D_{1}\right|>1+\left|T_{1}\right| \text { and }\left|D_{0}\right|>1+\left|T_{0}\right|
$$

then there exists $\alpha>1$ such that both affine maps of the border collision normal form are expansions in the metric $d_{\alpha}$.

Note that it is a simple exercise to show that

$$
\|x-y\|_{2} \leq d_{\alpha}(x, y) \leq \sqrt{1+\alpha^{2}}\|x-y\|_{2}
$$

and so if $d_{\alpha}(J x, J y) \geq \alpha d_{\alpha}(x, y)$ for some $\alpha>1$ then $d_{\alpha}\left(J^{k} x, J^{k} y\right) \geq \alpha^{k} d_{\alpha}(x, y)$ and so

$$
\left\|J^{k} x-J^{k} y\right\|_{2} \geq \frac{\alpha^{k}}{\sqrt{1+\alpha^{2}}}\|x-y\|_{2}
$$

and so there exists $N$ such that $\alpha^{k} / \sqrt{1+\alpha^{2}}>1$ for $k \geq N$, the $k^{\text {th }}$ iterates of the affine maps of the border collision normal form are expanding in the Euclidean metric for all $k \geq N$, and indeed, the same argument shows that if (12) holds then any $k$ products of the different Jacobians $(k \geq N)$ is also expanding in the Euclidean metric.

\section{Absolutely continuous invariant measures}

Invariant measures provide a way of describing the distribution of points under iteration over time. An invariant measure $\mu$ of a map $F$ has the property that $\mu(S)=\mu\left(F^{-1}(S)\right)$ for any measureable set $S$, and time averages converge to spatial averages for $\mu$-a.e. point $x$. If $m$ is the standard Lebesgue measure on $\mathbb{R}^{n}$ and $m(S)=0$ implies that $\mu(S)=0$ for all measureable sets $S$ then $\mu$ is absolutely continuous with respect to Lebesgue measure (often contracted to absolutely continuous). Note that not all sets of positive Lebesgue measure in $\mathbb{R}^{n}$ have topological dimension $n$ (think of fat fractals) so the existence of and absolutely continuous invariant measure does not imply that the support of the measure (i.e. the geometric attractor) contains open sets and hence has topological dimension $n$, although it does imply that the Hausdorff or fractal dimension is $n$.

This last claim follows from the observation that if the Hausdorff dimension of the attractor $S$ is strictly less than $n$ then $m(S)=0$ and so the absolute continuity of $\mu$ implies that $\mu(S)=0$ also. But if $S$ is the attractor with corresponding invariant measure $\mu$ then $\mu(S)=1$, a contradiction. Hence the Hausdorff dimension of $S$ is $n$. Although this means that the Hausdorff dimension is as large as it can be (since we are working in $\mathbb{R}^{n}$ ) this does not imply that $S$ is large from the point of view of Lebsgue measure - indeed, $S$ may have zero Lebesgue measure, as examples such as those in [Cabrelli et al., 2002] show.

A series of results on the existence of absolutely continuous invariant measures were proved around the turn of the century for different classes of piecewise expanding maps [Buzzi, 1999, 2001; Buzzi \& Keller, 2001; Saussol, 2000; Tsujii, 2001]. Since the border collision normal form is not piecewise expanding in 
the Euclidean metric, these results were not applied in this context, however the equivalent metric of the previous section now makes it possible to use some of these results in the context of the border collision normal form. Here we follow [Buzzi, 1999, 2001; Tsujii, 2001] as they seem most appropriate to our context.

A polyhedron is a region defined by the intersection of a finite set of closed half-planes. Tsujii [2001] uses the following formal setting for a set $\left\{T_{i}\right\}$ of affine maps defined on polyhedra $P_{i}, i=1, \ldots, M$. Let $U \subset \mathbb{R}^{n}$ be a polyhedron and $P_{i}, i=1, \ldots, M$ a set of polyhedra with disjoint interiors whose union is $U$ and for each $P_{i}$ let $T_{i}: P_{i} \rightarrow U$ be an affine injection into $U$. The collection $\left\{T_{i}\right\}_{i=1}^{M}$ is a piecewise expanding linear map if there is a metric in which each of the affine maps $T_{i}$ is expanding.

Theorem 2. [Tsujii, 2001; Buzzi, 2001] Every piecewise expanding linear map on a polyhedral domain admits an invariant measure which is absolutely continuous with respect to Lebesgue measure.

In fact [Tsujii, 2001], there are only finitely many such measures and all points in $U$ are in the basins of attraction of one of these measures. We will refer to Theorem 2 as Tsujii's Theorem in the remainder of this paper.

Corollary 1. If there exists an invariant polyhedral region $U$ for the $n$-dimensional border collision normal form and the inequalities (12) hold, then the border collision normal form has an attractor with an absolutely continuous invariant measure.

As remarked above, this is not enough to prove the existence of attractors with topological dimension equal to $n$. For this we require an alternative version of Theorem 2 due to Buzzi [1999, 2001]. The space of affine maps forms a vector space in the obvious way (using the matrices and the constants of two maps), and hence the set of border collision normal forms is a vector space (on the cross product of affine maps). If the affine pair is continuous on $x_{1}=0$, as is the case here, then the sum of two such maps is also continuous so the continuous pairs of affine maps form a vector space too. This means that notions of open and closed sets and hence Baire category can be applied to the border collision normal form, and a statement is true for almost all border collision normal forms if it holds on a residual set which is also positive Lebesgue measure in the vector space (see Buzzi [1999] for details). With these notions Buzzi [2001] proves results about the existence of absolutely continuous invariant measures for piecewise expanding linear maps similar to Tsujii's Theorem, but also gives extra information about the topological structure.

Theorem 3. [Buzzi, 2001] Every piecewise expanding linear map on a bounded polyhedral domain in $\mathbb{R}^{2}$ admits an invariant measure which is absolutely continuous with respect to Lebesgue measure and whose support contains open sets. Almost every expanding piecewise linear map on a bounded polyhedral domain in $\mathbb{R}^{n}, n>2$ admits an invariant measure which is absolutely continuous with respect to Lebesgue measure and whose support contains open sets.

Corollary 2. If there exists a region of parameter space $B_{2}$ such that for all parameters in $B_{2}$ the twodimensional border collision normal form has an invariant polyhedral region $U$ and the inequalities (12) hold, then for all parameters in $B_{2}$ the border collision normal form has an attractor with an absolutely continuous invariant measure and topological dimension equal to two.

\section{Back to the border collision normal form: $n=2$}

To apply the corollaries of the previous section it remains to show that there exists a compact invariant polyhedron for the border collision normal form with parameters constrained by (12). In two dimensions we will show that such a region exists by the simple rectangular construction shown in Figure 1: by choosing the corners appropriately it will be possible to obtain a set of inequalities defining an open region in the four dimensional parameter space such that this rectangle is invariant. Recall that we are working with (1), (2) and $\mu=1$.

Throughout this section we assume

$$
\begin{aligned}
& D_{0}<0, \quad D_{1}>0, \quad T_{0}<0, \quad T_{1}>0, \\
& \left|D_{0}\right|>1+\left|T_{0}\right|, \quad\left|D_{1}\right|>1+T_{1}
\end{aligned}
$$




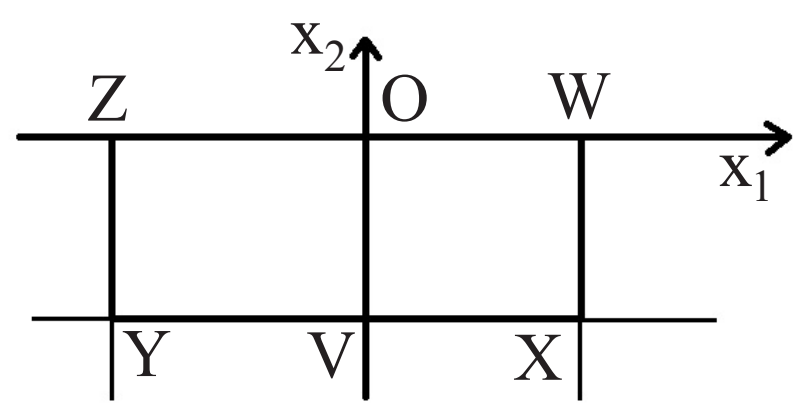

Fig. 1. Schematic diagram of the construction of the invariant region. $W$ and $X$ are on the line $x_{1}=\frac{\left|D_{0}\right|}{D_{1}\left(\left|D_{0}\right|-1\right)}, X, V$ and $Y$ lie on the line $x_{2}=-\frac{\left|D_{0}\right|}{\left|D_{0}\right|-1}$ and $Y$ and $Z$ on $x_{1}=-\frac{1}{\left|D_{0}\right|-1}$.

and

$$
D_{1}+\left|D_{0}\right|-D_{1}\left|D_{0}\right|>0
$$

The invariant region we construct (with no claim for optimality) is based on the points

$$
\begin{gathered}
W=\left(\frac{\left|D_{0}\right|}{D_{1}\left(\left|D_{0}\right|-1\right)}, 0\right), \quad X=\left(\frac{\left|D_{0}\right|}{D_{1}\left(\left|D_{0}\right|-1\right)},-\frac{\left|D_{0}\right|}{\left(\left|D_{0}\right|-1\right)}\right) \\
Y=\left(-\frac{1}{\left|D_{0}\right|-1},-\frac{\left|D_{0}\right|}{\left|D_{0}\right|-1}\right), \quad Z=\left(-\frac{1}{\left|D_{0}\right|-1}, 0\right)
\end{gathered}
$$

and the two intermediate points

$$
O=(0,0) . \quad V=\left(0,-\frac{\left|D_{0}\right|}{\left|D_{0}\right|-1}\right)
$$

The rectangle $W X Y Z$ is divided into two rectangles by the switching surface $x=0, O W X V$ and $O V Y Z$. These latter rectangles will play the role of the polyhedra $P_{i}$ in Tsujii's Theorem (Theorem 2 above) and the larger rectangle that of $U$. Note that since the two maps forming the border collision normal form are affine, if the images of the corners of the rectangles are mapped into the larger rectangle, then the larger rectangle is invariant $\left(T_{i}\left(P_{i}\right) \subseteq U\right)$.

Now, $F(O)=(1,0)$, and so, since (15) implies that $\frac{\left|D_{0}\right|}{D_{1}\left(\left|D_{0}\right|-1\right)}>1, F(0)$ is in $O W X V$. Similarly

$$
F(W)=\left(1+\frac{T_{1}\left|D_{0}\right|}{D_{1}\left(\left|D_{0}\right|-1\right)},-\frac{\left|D_{0}\right|}{\left|D_{0}\right|-1}\right)
$$

and so this is also in $O W X V$ provided

or

$$
1+\frac{T_{1}\left|D_{0}\right|}{D_{1}\left(\left|D_{0}\right|-1\right)}<\frac{\left|D_{0}\right|}{D_{1}\left(\left|D_{0}\right|-1\right)}
$$

$$
T_{1}<\frac{D_{1}+\left|D_{0}\right|-D_{1}\left|D_{0}\right|}{\left|D_{0}\right|}
$$

since this implies it lies on the line segment between $X$ and $V$.

Next, $F(X)$ lies on the line $Y X$, i.e. $x_{2}=-\frac{\left|D_{0}\right|}{\left|D_{0}\right|-1}$, with $x_{1}$-coordinate

$$
1+\frac{T_{1}\left|D_{0}\right|}{D_{1}\left(\left|D_{0}\right|-1\right)}-\frac{\left|D_{0}\right|}{\left|D_{0}\right|-1}=-\frac{1}{\left|D_{0}\right|-1}+\frac{T_{1}\left|D_{0}\right|}{D_{1}\left(\left|D_{0}\right|-1\right)} .
$$


The first of these expressions shows that it lies to the left of $F(W)$ and the second that it is to the right of $Y$, so there are no new constraints from this point. Finally, for the right hand rectangle $O W X V, F(V)=Z$. so there is no new constraint required here either.

The points $O$ and $V$ already have already been dealt with, so there remain only the two points $Y$ and $Z$ from the left hand region $O V Y Z$. The second coordinates of $F(Y)$ and $F(Z)$ show that they are both on the horizontal line $Y X ; F(Y)$ has $x_{1}$-coordinate

$$
1-\frac{T_{0}}{\left|D_{0}\right|-1}-\frac{\left|D_{0}\right|}{\left|D_{0}\right|-1}=-\frac{1}{\left|D_{0}\right|-1}-\frac{T_{0}}{\left|D_{0}\right|-1}
$$

the second of which explains the choice $T_{0}<0$, and the $x_{1}$-coordinate of $F(Z)$ is

$$
1-\frac{T_{0}}{\left|D_{0}\right|-1} \text {. }
$$

Thus $F(Z)$ is to the right of $F(Y)$ and both lie on the line between $Y$ and $X$ if $T_{0}<0$ (which is true by assumption) and

$$
1-\frac{T_{0}}{\left|D_{0}\right|-1}<\frac{\left|D_{0}\right|}{D_{1}\left(\left|D_{0}\right|-1\right)} .
$$

With a little rearranging this becomes

$$
0<-T_{0}<\frac{D_{1}+\left|D_{0}\right|-D_{1}\left|D_{0}\right|}{D_{1}} .
$$

With these calculations complete, we can state the main result of this section.

Theorem 4. Consider the border collision normal form with $\mu=1$ and suppose $\left(D_{0}, D_{1}, T_{0}, T_{1}\right)$ are chosen in the region $B_{2}$ defined by

$$
D_{0}<-1, \quad 1<D_{1}, \quad D_{1}+\left|D_{0}\right|-D_{1}\left|D_{0}\right|>0
$$

and

$$
0<T_{1}<\frac{D_{1}+\left|D_{0}\right|-D_{1}\left|D_{0}\right|}{\left|D_{0}\right|}, \quad 0<-T_{0}<\frac{D_{1}+\left|D_{0}\right|-D_{1}\left|D_{0}\right|}{D_{1}} .
$$

Then the border collision normal form has an attractor with an invariant measure absolutely continuous with respect to the two dimensional Lebesgue measure, and with non-empty interior.

Proof. Note that the third inequality of (19) is equivalent to $D_{1}<\left|D_{0}\right| /\left(\left|D_{0}\right|-1\right)$, the right hand side of which is greater than one and so $B_{2}$ is non-empty and open.

Consider the rectangle $W X Y Z$ defined at the beginning of this section. Gathering together the different inequalities above the statement of the theorem, this is invariant provided (14), (15), (17) and (18) hold, and by Corollary 2 of the previous section such an invariant region contains an attractor with non-empty interior and an absolutely continuous invariant measure if the inequalities (12) hold.

Now, inequalities (12) hold if (14) hold, which are clearly implied by (19) and (20), whilst (15) is implied by (19). Finally, (20) is essentially a restatement of (17) and (18) so these also hold and the result is proved.

The region $B_{2}$ is thus a region of parameter space on which Theorem 1 of the introduction holds with $n=2$.

\section{Higher dimensions}

Tsujii's Theorem (Theorem 2) holds in arbitrary dimension, $\mathbb{R}^{n}$. The $n$-dimensional border collision normal form was derived by di Bernardo [di Bernardo, 2003] and it is known that there are parameters for which the attractor has topological dimension $n$ [Glendinning \& Jeffrey, 2012]. Here we extend this result to an open set of parameter values in the case of Hausdorff deimension $n$, and show that Buzzi's Theorem 
(Thereom 3, [Buzzi, 2001]) holds. In $n$-dimensions then for typical continuous piecewise affine maps (1) the matrices $A_{j}, j=0,1$, of (1) can be chosen in observer canonical form [di Bernardo, 2003; di Bernardo et al., 2011]

$$
\left(\begin{array}{ccccc}
a_{1, j} & 1 & 0 & \ldots & 0 \\
a_{2, j} & 0 & 1 & \ldots & 0 \\
\vdots & \vdots & \vdots & \ddots & \vdots \\
a_{n-1, j} & 0 & 0 & \ldots & 1 \\
a_{n, j} & 0 & 0 & \ldots & 0
\end{array}\right)
$$

for constants $a_{r, j}$, and the constant vector is $(\mu, 0,0, \ldots, 0)^{T}$. As before we may take $\mu=1$ provided $\mu$ is non-zero. The natural generalization of the metric $d_{\alpha}$ on $\mathbb{R}^{2}$ defined in section 2 to $\mathbb{R}^{n}$ is

$$
d_{\alpha, n}(x, y)=\sum_{s=1}^{n} \alpha^{n-s}\left|x_{s}-y_{s}\right|, \quad \alpha>0
$$

(more subtle choices with different weights on different coordinates may optimize results). Then if $J$ is one of the matrices defined by (21) and $\delta_{s}=x_{s}-y_{s}$ then

$$
d_{\alpha, n}(J x, J y)=\left(\sum_{s=1}^{n-1} \alpha^{n-s}\left|\delta_{s+1}+a_{s, j} \delta_{s}\right|\right)+\left|a_{n, j}\right|\left|\delta_{1}\right| .
$$

By exactly the same argument as in section two,

$$
\begin{aligned}
d_{\alpha, n}(J x, J y) & \geq\left(\sum_{s=1}^{n-1} \alpha^{n-s}\left(\left|\delta_{s+1}\right|-\left|a_{s, j}\right|\left|\delta_{1}\right|\right)\right)+\left|a_{n, j}\right|\left|\delta_{1}\right| \\
& \geq \alpha\left(\alpha^{-1}\left|a_{n, j}\right|-\sum_{1}^{n-1} \alpha^{n-s-1}\left|a_{s, j}\right|\right)\left|\delta_{1}\right|+\alpha \sum_{2}^{n} \alpha^{n-s}\left|\delta_{s}\right| .
\end{aligned}
$$

Hence $d_{\alpha, n}(J x, J y) \geq \alpha d_{\alpha, n}(x, y)$ with $\alpha>1$, i.e. the $n$-dimensional map is expanding, if there exists $\alpha>1$ such that

$$
\alpha^{n-1}<\alpha^{-1}\left|a_{n, j}\right|-\sum_{1}^{n-1} \alpha^{n-s-1}\left|a_{s, j}\right|
$$

i.e. if

$$
\alpha^{n}+\left(\sum_{s=1}^{n-1}\left|a_{s, j}\right| \alpha^{n-s}\right)-\left|a_{n, j}\right|<0
$$

Such $\alpha$ exist provided the left hand side of (22) is negative at $\alpha=1$, so the map is piecewise expanding if

$$
\left|a_{n, j}\right|>1+\sum_{s=1}^{n-1}\left|a_{s, j}\right|, \quad j=0,1 .
$$

Tsujii's Theorem therefore implies the following $n$-dimensional result.

Theorem 5. Consider the border collision normal form in $\mathbb{R}^{n}, n>2$, with $\mu=1$. If there is an open region of parameter space $B_{n}$ such that for all parameters in $B_{n}$ the $n$-dimensional border collision normal form has an invariant polyhedron and (23) holds then the invariant polyhedron contains an attractor with an invariant measure absolutely continuous with respect to (the $n$-dimensional) Lebesgue measure and with Hausdorff dimension equal to $n$.

For this result to have content it remains to prove that there exist parameters which satisfy the conditions of the theorem, and in particular the existence of an invariant polyhedron for parameters satisfying (23).

The construction is very similar to the construction in the two-dimensional case described in section three. First, some notation and initial assumptions. Let

$$
-D_{0}=a_{n, 0}>0, \quad D_{1}=-a_{n, 1}>0
$$


and assume

$$
a_{r, 0}<0 \quad \text { and } \quad a_{r, 1}>0, \quad r=1, \ldots, n-1 .
$$

Define

$$
b_{r, 0}=-D_{1} a_{r, 0} /\left|D_{0}\right|, \quad r=1, \ldots, n-1
$$

and the partial sums

$$
S(p, 0)=-\sum_{i=p}^{n-1} b_{i, 0}, \quad S(p, 1)=\sum_{i=p}^{n-1} a_{i, 1}
$$

so $S(p, j)>0, j=0,1$, and let

$$
M(p)=\sum_{i=p}^{n-1} \max \left\{b_{i, 0}, a_{i, 1}\right\} .
$$

for $p=1, \ldots, n-1$. The definition of the coefficients $b_{r, 0}$ introduces an asymmetry between the treatment of the cases $j=0$ and $j=1$ which could be avoided, but it makes some of the calculations in the proof of the theorem below rather simpler.

Theorem 6. Consider the $n$-dimensional border collision normal form $F$ with $\mu=1$ and $n>2$. Let $B_{n}$ be the region of parameter space on which the coefficients of the border collision normal form satisfy (24) and (25) together with $\left|D_{0}\right|>1+D_{1} S(1,0) /\left|D_{0}\right|, D_{1}>1+S(1,1)$, and

$$
M(1)<\frac{D_{1}+\left|D_{0}\right|-D_{1}\left|D_{0}\right|}{\left|D_{0}\right|} .
$$

Then $F$ is piecewise expanding and there is an invariant polyhedron.

Hence the conditions of Theorems 5 holds with $n>2$.

Proof. Let $F$ denote the $n$-dimensional border collision normal form. $F$ is piecewise expanding in the metric $d_{\alpha, n}$ for some $\alpha>1$ since the two inequalities for $\left|D_{0}\right|$ and $D_{1}$ in the statement of the theorem are equivalent to (23).

Consider the rectangle defined by the following inequalities:

$$
\begin{aligned}
& -\frac{1}{\left|D_{0}\right|-1} \leq x_{1} \leq \frac{\left|D_{0}\right|}{D_{1}\left(\left|D_{0}\right|-1\right)} \\
& -\frac{\left|D_{0}\right|}{\left|D_{0}\right|-1} \leq x_{r} \leq \frac{M(r)\left|D_{0}\right|}{D_{1}\left(\left|D_{0}\right|-1\right)}
\end{aligned}
$$

$r=2, \ldots n-1$, and

$$
-\frac{\left|D_{0}\right|}{\left|D_{0}\right|-1} \leq x_{n} \leq 0
$$

This rectangle, which we denote by $U$, can be divided into two closed rectangles $P_{0}$ in $x_{1} \leq 0$ and $P_{1}$ in $x \geq 0$, intersecting on the hyperplane $x_{1}=0$. We will show that $F\left(P_{i}\right) \subseteq U, i=0,1$. so

Consider $x \in P_{1}$ with $x_{1} \geq 0$, so $0 \leq x_{1} \leq \frac{\left|D_{0}\right|}{D_{1}\left(\left|D_{0}\right|-1\right)}$. Then the $x_{n}$-coordinate, $x_{n}^{\prime}$, of $F(x)$ is $-D_{1} x_{1}$,

$$
-\frac{\left|D_{0}\right|}{\left|D_{0}\right|-1} \leq x_{n}^{\prime} \leq 0
$$

as required, cf. (30). Similarly, the $x_{n-1}$-coordinate, $x_{n-1}^{\prime}$, of $F(x)$ is $a_{n-1,1} x_{1}+x_{n}$ and since $a_{n-1,1}=$ $S(n-1,1), 0 \leq x_{1} \leq \frac{\left|D_{0}\right|}{D_{1}\left(\left|D_{0}\right|-1\right)}$ and $-\frac{\left|D_{0}\right|}{\left|D_{0}\right|-1} \leq x_{n} \leq 0$, this implies

$$
-\frac{\left|D_{0}\right|}{\left|D_{0}\right|-1} \leq x_{n-1}^{\prime} \leq S(n-1,1) \frac{\left|D_{0}\right|}{D_{1}\left(\left|D_{0}\right|-1\right)}
$$


and so the $x_{n-1}$-coordinate of the image also lies in the range specified by $U$, cf. (29).

In general, if $r=2,3, \ldots, n-1$, then

$$
-\frac{\left|D_{0}\right|}{\left|D_{0}\right|-1} \leq x_{r}^{\prime} \leq\left(M(r+1)+a_{r, 1}\right) \frac{\left|D_{0}\right|}{D_{1}\left(\left|D_{0}\right|-1\right)}
$$

and so, since $M(r) \geq M(r+1)+a_{r, 1}$ this satisfies the constraints defining $U$.

Finally the $x_{1}$-coordinate, $x_{1}^{\prime}$, of $F(x)$ is $a_{1,1} x_{1}+x_{2}+1$ which is $S(1,1) x_{1}+x_{2}+1$ and so

$$
-\frac{\left|D_{0}\right|}{\left|D_{0}\right|-1}+1=-\frac{1}{\left|D_{0}\right|-1} \leq x_{1}^{\prime} \leq 1+M(1) \frac{\left|D_{0}\right|}{D_{1}\left(\left|D_{0}\right|-1\right)}
$$

and so this also is in the range of values defining $U$ provided $1+M(1) \frac{\left|D_{0}\right|}{D_{1}\left(\left|D_{0}\right|-1\right)}<\left|D_{0}\right| /\left(D_{1}\left(\left|D_{0}\right|-1\right)\right)$, i.e.

$$
M(1)<\frac{D_{1}+\left|D_{0}\right|-D_{1}\left|D_{0}\right|}{\left|D_{0}\right|},
$$

an inequality which holds due to the constraint on $M(1)$ in the statement of the theorem.

The proof that $F\left(P_{0}\right) \subseteq U$ is almost precisely the same. First consider $x_{n}$. The new coordinate has $x_{n}^{\prime}=\left|D_{0}\right| x_{1}$ and since $-1 /\left(\left|D_{0}\right|-1\right) \leq x_{1} \mid \leq 0$ in $P_{0},-\left|D_{0}\right| /\left(\left|D_{0}\right|-1\right) \leq x_{n}^{\prime} \leq 0$ as required.

Now if $r \in\{2, \ldots, n-1\}, x_{r}^{\prime}=a_{r, 0} a_{1}+x_{r+1}=-\left|D_{0}\right| b_{r, 0} x_{1} / D_{1}+x_{r+1}$. The maximum this can be is obtained by choosing the most negative value of $x_{1}$ the maximum of $x_{r+1}$, which gives

$$
\frac{\left|D_{0}\right| b_{r, 0}}{D_{1}\left(\left|D_{0}\right|-1\right)}+\frac{M(r+1)\left|D_{0}\right|}{D_{1}\left(\left|D_{0}\right|-1\right)} \leq M(r) \frac{\left|D_{0}\right|}{D_{1}\left(\left|D_{0}\right|-1\right)}
$$

as required, and the minimum is just the minimum of $x_{r+1}$, i.e $-\left|D_{0}\right| /\left(D_{1}\left(\left|D_{0}\right|-1\right)\right)$ as required. Finally note that the smallest $x_{1}^{\prime}$ can be is the smallest value of $x_{2}+1$ as in the case of $P_{1}$, whilst the largest is less than or equal to $1+M(1) \frac{\left|D_{0}\right|}{D_{1}\left(\left|D_{0}\right|-1\right)}$ which leads to the same condition (31) as before.

To complete the proof of Theorem 1 we need to show that the generic result of Buzzi [2001] (Theorem 3) is also generic for maps in observer canonical form $(21)$ in $B_{n}$.

Buzzi's results hold in the space of piecewise affine maps with switching surface $x_{1}=0$. These are parametrized by the three quantities $\left(A_{0}, A_{1}, m\right)$ of $(1)$. There is a simple equivalence class that can be defined on this space, where two triples $\left(A_{0}, A_{1}, m\right)$ and $\left(A_{0}^{\prime}, A_{1}^{\prime}, m^{\prime}\right)$ are equivalent iff there exists a constant $n \times n$ invertible matrix $U$ whose first row is $\left(\begin{array}{lllll}1 & 0 & 0 & \ldots & 0\end{array}\right)$ and a constant vector $c$ with first component equal to zero such that

$$
A_{0}^{\prime}=U A_{0} U^{-1}, \quad A_{1}^{\prime}=U A_{1} U^{-1}, \quad m^{\prime}=U m_{0}+c-U A_{0} U^{-1} c .
$$

This is equivalent to saying that the two systems are equivalent under the change of coordinate $y=U x+c$ with $x_{1}=0$ being equivalent to $y_{1}=0$; the form of $m^{\prime}$ looks dependent on $A_{0}$, but is equal to the equivalent expression with $A_{0}$ replaced by $A_{1}$ using the continuity condition across $x_{1}=0$.

If two piecewise affine maps are equivalent under this equivalence relation then the following properties are common to both or to neither as they are preserved by affine changes of coordinates: they have an invariant polyhedral domain; they are piecewise expanding; they have an attractor with topological dimension $n$, i.e. an attractor that contains open sets.

Buzzi's Theorem (Theorem 3) holds for maps which are general continuous piecewise affine maps with switching surface $x_{1}=0$; we will say such maps are in $\mathcal{N}_{0}$. To show the results hold in the space $\mathcal{N}_{2}$ of border collision normal forms we will also have to consider the set of maps $\mathcal{N}_{1} \subset \mathcal{N}_{0}$ which are in the same equivalence class as a map in $\mathcal{N}_{2}$.

Now, a property that is generic in $\mathcal{N}_{0}$ is generic in $\mathcal{N}_{1}$ since by [di Bernardo, 2003; di Bernardo et al., 2011] the set of piecewise affine maps that cannot be brought into this form is determined by the vanishing of the determinants of two matrices each of which can be written as a polynomial condition on the parameters and hence has zero measure [Buzzi, 1999] (or full measure, but this is clearly not the case). Hence the property of having an attractor containing open sets is generic in $\mathcal{N}_{1}$ and hence in $\mathcal{N}_{2}$. This completes the proof of Theorem 1. 


\section{Conclusion}

In this paper we have shown that there are open sets of parameter values for which the $n$-dimensional border collision normal form has an attractor with fractal dimension equal to $n$ and, if $n=2$, topological dimension also equal to two. Moreover, if $n>2$ then these attractors have topological dimension equal to $n$ for generic maps. This result relies on the use of an equivalent metric in which each map defining the border collision normal form is expanding (as they are not expanding in the standard Euclidean metric). The equivalent metric used is essentially that introduced in [Glendinning, 2014] to deal with a similar issue in the contracting case. The results also lend weight to the argument of Glendinning and Jeffrey [2012] that there is a curse of dimensionality in the study of piecewise smooth bifurcation theory. The results presented here show that there are open sets of parameter values for which the $n$-dimensional border collision normal form has an attractor that cannot exist in the $(n-1)$-dimensional normal form. This suggests that bifurcation theorems in the piecewise smooth case depend on the dimension of the phase space. This is in marked contrast with the smooth case, where results such as the centre manifold theorem show that the dimension of the phase space contributes only extra hyperbolic directions in codimension one local bifurcations.

The border collision normal form studied here is derived via a Taylor expansion argument, and in applications the true model is a perturbation of the affine maps, in which the nonlinear terms have been re-introduced. Although there are results for piecewise smooth maps in the literature (e.g. [Buzzi, 2000; Tsujii, 2000]) they require greater smoothness than is appropriate in the context of piecewise smooth bifurcations (for example in the grazing-sliding bifurcation the correction terms are order $|x|^{\frac{3}{2}}$ [di Bernardo et al., 2001, 2008; Glendinning \& Jeffrey, 2012]). This remains an important outstanding issue.

The parameter regions defined in the proofs of the theorems were chosen for ease of calculation rather than completeness. A fuller description of those parameters which have attractors of different topological dimensions would be worthwhile, It is also worth noting that Tsujii's Theorem does not apply directly to the parameters used in [Glendinning \& Wong, 2011], where the proof is driven by the existence of a finite Markov partition. It seems likely that in this and other examples Tsujii's Theorem will apply to an iterate of the map, i.e. the map is not piecewise expanding, but some higher iterate is. In this case the conclusion of Tsujii's Theorem still holds, but more effort is required to prove that the map is eventually piecewise expanding.

\section{Acknowledgments}

I am grateful to Masato Tsujii for clarifying the role of different metrics in his results. This work was partially funded by EPSRC Mathematics Platform Engagement grant EP/I01912X/1.

\section{References}

Banerjee, S. \& Grebogi, C. [1999] "Border Collision Bifurcations in Two-Dimensional Piecewise Smooth Maps," Phys. Rev. E 59, 4052-4061.

Banerjee, S., Ranjan, P. \& Grebogi, C. [2000] "Bifurcations in two-dimensional piecewise smooth maps theory and applications in switching circuits," IEEE Trans. Circ. Syst., I: Fundam. Theory Appl. 47, 633-643.

Banerjee, S., Yorke, J.A. \& Grebogi, C. [1998] "Robust Chaos" Phys. Rev. Lett. 80, 3049-3052.

Barnsley, M.F., Elton, J., Hardin, D. \& Massopust, P. [1989] "Hidden variable fractal interpolation functions," SIAM J. Math. Anal. 20, 1218-1242.

di Bernardo. M. [2003] "Normal forms of border collision in high dimensional non-smooth maps," Proceedings IEEE ISCAS 2003 3, 76-79.

di Bernardo, M., Budd, C.J., \& Champneys, A.R. [2001] "Grazing and Border-Collision in PiecewiseSmooth Systems: A Unifed Analytical Framework," Phys. Rev. Lett. 86, 2554-2556.

di Bernardo, M., Budd, C.J., Champneys, A.R. \& Kowalczyk, P. [2008] Piecewise-smooth Dynamical Systems: Theory and Applications, (Springer, London).

di Bernardo, M., Montanaro, U. \& Santini, S. [2011] "Canonical Forms of Generic Piecewise Linear Continuous Systems," IEEE Trans. Automatic Control 56, 1911-1915. 
Bischi, G-I., Mammana, C. \& Gardini, L. [2000] "Multistability and cyclic attractors in duopoly games," Chaos, Sol. \& Fract. 11, 543-564.

Buzzi, J. [1999] "Absolutely continuous invariant measures for generic multi-dimensional piecewise affine expanding maps," Int. J. Bifn. \& Chaos 9, 1743-1750.

Buzzi, J. [2000] "A.C.I.M.s for arbitrary expanding piecewise $\mathbb{R}$-analytic mappings of the plane," Ergod. Thy. \& Dyn. Syst. 20, 697-708.

Buzzi, J. [2001] "Thermodynamic formalism for piecewise invertible maps: Absolutely continuous invariant measures as equilibrium states," in Smooth Ergodic Theory and Its Applications, eds. Katok, A., de la Llave, R., Pesin, Y. \& Weiss, H. AMS Proc. Symp. Pure Math 69, 749-784.

Buzzi, J. \& Keller, G. [2001] "Zeta functions and transfer operators for multidimensional piecewise affine and expanding maps," Ergodic Thy. E Dyn. Syst. 21, 689-716.

Cánovas, J.S. \& Lineros, A. [2001] "Topological dynamic classification of duopoly games," Chaos, Solit. \& Fract. 12, 1259-1266.

Cabrelli, C.A., Hare, K.E. \& Molte, U.M. [2002] "Sums of Cantor sets yielding an interval," J. Aust. Math. Soc. 73, 405-418.

Dobrynskii, V.A. [1998] "The existence of two-dimensional topologically mixed attractors for some piecewise linear maps of the plane," Izv. Math. 62, 53-58.

Dobrynskiy, V.A. [1999] "On attractors of piecewise linear 2-endomorphisms," Nonl. Anal. 36, 423-455.

Gardini, L. [1992] "Some global bifurcations of two-dimensional endomorphisms by use of critical lines," Nonl. Anal. 18, 361-399.

Glendinning, P. [2014] "The border collision normal form with stochastic switching surface," SIAM. J. Appl. Dynam. Syst. 13, 181-193.

Glendinning, P. \& Jeffrey, M. [2012] "Grazing-sliding bifurcations, the border collision normal form, and the curse of dimensionality for nonsmooth bifurcation theory," preprint.

Glendinning, P. \& Wong, C.H. [2011] "Two-dimensional attractors in the border-collision normal form," Nonlinearity 24, 995-1010.

Ishii, Y. \& Sands, D. [2007] "Lap number entropy formula for piecewise affine and projective maps in several dimensions," Nonlinearity 20, 2755-2772.

Lozi, R. [1978] "Un attracteur étrange (?) du type attracteur de Hénon," J. Physique (Paris) 39, C5 9-10.

Mira, C., Gardini, L., Barugola, A. \& Cathala, J.C. [1996] Chaotic Dynamics in Two-Dimensional Noninvertible Maps, (World Scientific, Singapore).

Mira, C., Rauzy, C., Maistrenko, Y. \& Sushko, I. [1996] "Some properties of a two-dimensional piecewiselinear noninvertible map," Int. J. Bif. \& Chaos 6, 2299-2319.

Misiurewicz, M. [1980] "Strange attractors for the Lozi mappings," Ann. N.Y. Acad. Sci. 357, 348-358.

Nusse, H.E., Ott, E. \& Yorke, J.A. [1994] "Border-collision bifurcations: An explanation for observed bifurcation phenomena," Phys. Rev. E 49, 1073-1076.

Nusse, H.E. \& Yorke, J.A. [1992] "Border-collision bifurcation including 'period two to period three' for piecewise smooth systems," Physica D 57, 39-57.

Saussol, B. [2000] "Absolutely continuous invariant measures for multidimensional expanding maps," Israel Journal of Mathematics 116, 223-248.

Simpson, D.J.W. [2010] Bifurcations in Piecewise-Smooth Continuous Systems,. (World Scientific, Singapore).

Simpson, D.J.W. \& Meiss, J.D. [2010] "Resonance near Border-Collision Bifurcations in Piecewise-Smooth, Continuous Maps," Nonlinearity 23, 3091-3118.

Tsujii, M. [2000] "Abolutely continuous invariant measures for piecewise real-analytic expanding maps on the plane," Commun. Math. Phys. 208, 605-622.

Tsujii, M. [2001] "Absolutely continuous invariant measures for expanding piecewise linear maps," Invent. Math. 143, 349-373.

Young, L.S. [1985] "Bowen-Ruelle measures for certain piecewise hyperbolic maps," Trans. Amer. Math. Soc. 287, 41-48. 